\title{
Resilience as a Possible Predictor for Psychological Distress in Chronic Spinal Cord Injured Patients Living in the Community
}

\author{
Jung-In Shin, M.D., Jeong-Ho Chae, M.D., Ph.D. ${ }^{1}$, Jung-Ah Min, M.D. ', Chang-Uk Lee, M.D., Ph.D. ', \\ Sung-Il Hwang, M.D., Bum-Suk Lee, M.D., Sang-Hoon Han, M.D., Hye-In Ju, M.D., Cha-Yeon Lee \\ Department of Rehabilitation Medicine, National Rehabilitation Hospital, Seoul 142-884, ${ }^{1}$ Department of Psychiatry, \\ Seoul St. Mary's Hospital, The Catholic University of Korea College of Medicine, Seoul 137-701, Korea
}

Objective To investigate whether higher resilience level predicts low levels of psychological distress in chronic SCI patients living in the community.

Method Thirty seven patients (mean age 41.5 \pm 10.9 , male : female=28 : 9) with chronic spinal cord injury (duration $8.35 \pm 7.0$ years) living in the community are included, who were hospitalized for annual checkups from November, 2010 to May, 2011. First, their spinal cord injury level, completeness and complications were evaluated. The patients completed questionnaires about their educational status, religion, employment status, marital status, medical and psychological history and also the following questionnaires: Hospital Anxiety and Depression Scale (HADS), ConnorDavidson Resilience Scale (CD-RISC), Alcohol Use Disorders Identification Test-alcohol consumption questions (AUDIT-C) and Health-related quality of life (EQ-5D). The patients were divided into two subgroups: patients with HADS $\geq 13$ are classified as high psychological distress group and others as low psychological distress group. We compared the two groups to find statistically significant differences among the variables.

Results CD-RISC, EQ-5D and employment status are significantly different between two groups ( $<<0.05)$. In a forward stepwise regression, we found that EQ-5D had a greater contribution than CD-RISC to the psychological distress level.

Conclusion In addition to health-related quality of life, resilience can be suggested as a possible predictor of psychological distress in chronic SCI patients.

Key Words Resilience, Predictor, Spinal cord injuries

Received May 2, 2012; Accepted August 28, 2012

Corresponding author: Jeong-Ho Chae

Department of Psychiatry, Seoul St. Mary's Hospital, The Catholic University of Korea College of Medicine, 505, Banpo-dong, Seocho-gu, Seoul 137-701, Korea

Tel: +82-2-2258-5443, Fax:+82-2-594-3870, E-mail: alberto@catholic. ac.kr

() This is an open-access article distributed under the terms of the Creative Commons Attribution Non-Commercial License (http:// creativecommons.org/licenses/by-nc/3.0) which permits unrestricted noncommercial use, distribution, and reproduction in any medium, provided the original work is properly cited.

Copyright $\odot 2012$ by Korean Academy of Rehabilitation Medicine

\section{INTRODUCTION}

Spinal cord injury (SCI) is one of the most disastrous injuries, often causing mental health problems which contribute to increased disability and reduced quality of life in such patients. Nearly half of the population with SCI suffered mental health problems of depression, anxiety, clinical-level stress or posttraumatic stress disorder. ${ }^{1}$ Previous studies have mainly focused on the measure- 
ment of depression or anxiety, risk factors of depression in spinal cord injuries or prevention of suicide attempts.

However, while some patients suffer from depression or other mental health problems, others adjust well to life after spinal cord injuries. Therefore, there is a need to focus on the well-adjusted patients, not the patients with psychological disorders, and to find the factors important in their adjustment. This is in line with the concept of resilience.

Psychological resilience embodies the personal qualities that enable one to thrive in the face of adversity. Resilience is also viewed as successful stress-coping ability. ${ }^{2}$ While many studies have been conducted of depression or other mental health problems in patients with SCI, studies of resilience in SCI are limited. Prior studies report a buffering effect of resilient characteristics against the perceptions of stress on depressive symptoms ${ }^{3}$ and found significant associations between resilience, satisfaction with life, spirituality, and depressive symptoms. ${ }^{4}$ Researchers agree that resilience is related to depressive symptoms and thus rehabilitation outcomes, and interventions based on resilience would be helpful. Therefore, this study was performed to investigate whether higher resilience level predicts low levels of psychological distress in SCI patients living in the community.

\section{MATERIALS AND METHODS}

\section{Patients}

A total of 37 patients who were hospitalized for annual checkups were recruited from November, 2010 to May, 2011. Each subject underwent clinical evaluation to determine his or her SCI level, completeness and complications. Neurologic injury was classified using the International Standards for Neurological Classification of SCI developed by the American Spinal Injury Association. For complications, pressure ulcer, uncontrolled spasticity, urinary tract infections, pain or respiratory problem for the last year were examined. All examinations were conducted by rehabilitation doctors. Patients with a past history of psychological illness were excluded. Written informed consent for this research was obtained from all participants. The study protocol was reviewed and approved by The Catholic University of Korea Institutional Review Board (IRB No.KC09FZZZ0211).

\section{Questionnaire}

The patients completed questionnaires about their educational status, religion, employment status, marital status, medical and psychological history.

The patients also completed the following questionnaires; Hospital Anxiety and Depression Scale (HADS), Connor-Davidson Resilience Scale (CD-RISC), Alcohol Use Disorders Identification Test-alcohol consumption questions (AUDIT-C) and Health-related quality of life (EQ-5D).

The HADS consists of two 7-item self-report subscales designed to assess current depressive and anxiety symptomatology in non-psychiatric hospital settings. Possible scores on the HADS range from 0 to 21 and the HADS has well-validated psychometric properties. ${ }^{5}$

Connor-Davidson Resilience Scale (CD-RISC) contains 25 items, all of which carry a 5-point range of responses, as follows: not true at all (0), rarely true (1), sometimes true (2), often true (3), and true nearly all of the time (4). The scale is rated based on how the subject has felt over the past month. The total score ranges from $0-100$, with higher scores reflecting greater resilience. ${ }^{2}$

Alcohol Use Disorders Identification Test is a tool of screening for alcohol dependence as well as for less severe alcohol problems. ${ }^{6}$ AUDIT-C is the short version of AUDIT consisting of 3 consumption items, and is known to be equal in accuracy of the full AUDIT. ${ }^{6}$

EQ-5D is a well-established and widely-used generic instrument for assessing health-related quality of life. ${ }^{7}$ The respondent classifies his/her prevailing state of health by selecting one of three different levels of problem severity within each of five health domains. ${ }^{8}$ The levels are none, moderate and severe/extreme (coded 1 through 3, respectively), while the domains are mobility, capacity for self-care, conduct of usual activities, pain/ discomfort and anxiety/depression.

\section{Analysis}

Statistics were performed using the Statistical Package for Social Sciences (SPSS) version 10.0 (SPSS Inc., Chicago, USA). The study group was divided into two subgroups with different levels of psychological distress; Patients with HADS $\geq 13$ were classified as high psychological distress group and others as low psychological distress group. Woolrich et al. ${ }^{9}$ presented the normative data of HADS in Chronic SCI patients at his study with 963 patients with mean duration of 19.5 years. The score 
was 12.3 for total, and 12.7 and 12.2 for tetraplegia and paraplegia, respectively. From this data, high psychological distress group was defined as HADS by a cut-off score of $\geq 13$. We compared the two subgroups to find statistically significant difference among the variables.

The total scores of questionnaires, time elapsed since injury, age at the moment of the SCI, current age and the period of education were compared with a t-test. Chi-square test was used to compare the distribution of gender, marital status, religion, employment status, SCI level and the presence of complications. The variables significantly different between the two groups in univariate analysis were entered into a logistic regression model to investigate the independent predictive value. Forward stepwise procedure was used due to significant intercorrelation between variables and multicollinearity. Null hypotheses of no difference were rejected if $\mathrm{p}$-values were less than 0.05 .

\section{RESULTS}

Of the 37 patients with chronic SCI, $76 \%$ were male and
$24 \%$ were female. Their mean age was 41.5 years. There were 17 patients (18.9\%) with complete tetraplegia, 6 patients $(16.2 \%)$ with incomplete tetraplegia, 11 patients (29.7\%) with complete paraplegia and 3 patients (8.1\%) with incomplete paraplegia.

Based on the cutoff point of 12 in total HADS score, 15 and 22 patients were designed as the low and the high psychological distress group, respectively.

We compared the demographic, clinical and psychological characteristics of the two subgroups with low and high psychological distress level. Demographic factors did not differ significantly by distress level except employment status. By comparing clinical factors, SCI levels, complications from the injury, time elapsed since injury and age at the moment of the SCI did not differ significantly between subgroups (Table 1).

Among tested psychological variables, CD-RISC and EQ-5D were significantly different between the two subgroups. The subgroup with a high psychological distress level had lower CD-RISC scores $(\mathrm{p}=0.009)$, and higher EQ-5D scores $(\mathrm{p}<0.001)$ than subgroup with a low psychological distress level. AUDIT-C did not significantly

Table 1. Demographic and Clinical Characteristics between the Subgroups with Different Levels of Psychological Distress

\begin{tabular}{|c|c|c|c|c|}
\hline & & $\begin{array}{c}\text { Low psychological distress } \\
\text { group }(n=15)\end{array}$ & $\begin{array}{l}\text { High psychological distress } \\
\text { group }(n=22)\end{array}$ & p-value \\
\hline \multicolumn{5}{|l|}{ Demographic variables } \\
\hline Age & & $38.5 \pm 10.5$ & $43.6 \pm 10.8$ & 0.171 \\
\hline \multirow[t]{2}{*}{ Sex } & Female & 4 & 4 & 0.538 \\
\hline & Male & 11 & 18 & \\
\hline \multirow[t]{2}{*}{ Employment status } & Employed & 9 & 6 & $0.047^{*}$ \\
\hline & Unemployed & 6 & 16 & \\
\hline \multirow[t]{2}{*}{ Religion } & Yes & 8 & 12 & 0.942 \\
\hline & No & 7 & 10 & \\
\hline \multirow[t]{2}{*}{ Marital status } & Spouse (-) & 10 & 18 & 0.465 \\
\hline & Spouse (+) & 5 & 4 & \\
\hline Education period & & $13.8 \pm 2.6$ & $13.2 \pm 2.7$ & 0.525 \\
\hline \multicolumn{5}{|l|}{ Clinical variables } \\
\hline \multirow[t]{2}{*}{ Level } & Tetraplegia & 7 & 14 & 0.306 \\
\hline & Paraplegia & 8 & 8 & \\
\hline \multirow[t]{2}{*}{ Complication } & Yes & 11 & 18 & 0.538 \\
\hline & No & 4 & 4 & \\
\hline Duration (month) & & $97.4 \pm 74.9$ & $102.1 \pm 88.5$ & 0.866 \\
\hline Age at onset & & $30.4 \pm 11.8$ & $35.0 \pm 12.5$ & 0.268 \\
\hline
\end{tabular}

${ }^{*} \mathrm{p}<0.05$ 
differ (Table 2). In a forward stepwise regression, we tried to estimate the independent influence as predictor for psychological distress, of CD-RISC and EQ-5D. We found that EQ-5D made a greater contribution than CD-RISC to psychological distress level, shown as B coefficients entailed at Table 3 .

\section{DISCUSSION}

The major aim of this study was to find whether resilience is helpful to predict psychological distress in chronic SCI patients living in the community. Of all the demographic and clinical factors, health related quality of life was the strongest predictor of psychological distress. Additionally, resilience was considered as a possible predictor but it did not reach statistical significance in logistic regression modeling.

Demographic factors except employment had no significant differences between the two subgroups. The patients with jobs showed lower distress level. Reintegration into work is considered to be an important goal of rehabilitation, and gives patients social status and meaning to life, enabling them to be financially independent. ${ }^{10}$ Thus,

Table 2. Comparison of Subgroups with Different Levels of Psychological Distress Regarding Psychological Characteristics

\begin{tabular}{lccc}
\hline & $\begin{array}{c}\text { Low psycho- } \\
\text { logical distress } \\
\text { group (n=15) }\end{array}$ & $\begin{array}{c}\text { High psycho- } \\
\text { logical distress } \\
\text { group (n=22) }\end{array}$ & p-value \\
\hline CD-RISC & $68.2 \pm 17.7$ & $50.2 \pm 18.1$ & $0.009^{*}$ \\
EQ-5D & $9.2 \pm 1.8$ & $11.6 \pm 1.8$ & $<0.001^{*}$ \\
AUDIT-C & $3.9 \pm 3.5$ & $2.9 \pm 3.3$ & 0.401 \\
\hline
\end{tabular}

CD-RISC: Connor-Davidson Resilience Scale, EQ-5D: Health-related quality of life, AUDIT-C: Alcohol consumption questions

${ }^{*} \mathrm{p}<0.05$ our finding that being employed is related to distress level is reasonable. This result was somewhat different from that of Schonherr et al. ${ }^{10}$ Life satisfaction in Schonherr's study was not associated with being employed. A reduced quality of life was particularly associated with an unsatisfactory vocational situation. Attention to vocational satisfaction issues has the potential to improve life satisfaction following SCI and should be included in further studies. In addition, employment is associated with EQ-5D in our study. Heath-related quality of life includes evaluation of self-care, mobility and activities. As a result, vocational satisfaction rather than being employed is important to psychological distress. Employed status can reinforce health-related quality of life, potentially lowering the psychological distress level.

Health related quality of life, as well as resilience, was more significant to the level of psychological distress of SCI patients than variables related to objective physical disabilities in this study. The SCI level, complication, time elapsed since injury and age at the moment of the SCI all did not differ between the subgroups. However, the study of Shin et al., ${ }^{11}$ of acute patients within 6 months after the injury, found motor complete injuries significantly affected depression, QOL, and stress. Migliorini et al., ${ }^{1}$ found three variables significantly impacted the likelihood of psychopathology: health, level of injury, and time since injury. Improvement in health decreased the likelihood of the presence of psychopathology. Each year since injury decreased the likelihood of the presence of psychopathology to the order of $2.6 \%$. Moreover, those with complete quadriplegia were more than threefold as likely not to suffer psychopathology compared with any other level of injury. In contrast to these studies, our study shows no significance of these variables. The difference is thought be caused by the time since injury. The mean duration of our study was 8.35 years and the participants were within 6 months from onset in the

Table 3. Predictor Weights of Regression Model Predicting Psychological Distress

\begin{tabular}{llcccc}
\hline & & B coefficient & Standard error & p-value & Exp (B) \\
\hline Step 1 & EQ-5D & 0.846 & 0.322 & 0.009 & 0.015 \\
& Constant & -8.129 & 3.347 & 0.035 & 0.000 \\
Step 2 & EQ-5D & 0.670 & 0.318 & 0.069 & 0.955 \\
& CD-RISC & -0.085 & 0.047 & 0.818 & 0.375 \\
\hline
\end{tabular}

Exp (B): Exponential value of B coefficient 
study of Shin et al. ${ }^{11}$ From the cross-national study of 355 community residing persons with SCI, who had lived with their lesion for 5 years or more reported more use of Acceptance coping than persons who had lived 1-4 years with their lesion. ${ }^{12}$ Coping strategies are conscious efforts to manage or reduce the stressful experiences in significance with chronic SCI patients. ${ }^{13}$ This can make a difference of psychopathology upon the onset duration. As Migliorini et al. ${ }^{1}$ mentioned as limitations, distribution according to the level of injury and time since injury was not equal in his study. Those with incomplete injuries were injured significantly more recently compared to those with complete injuries. A sample bias was made by a healthy survivor effect. In our study, the mean duration was 8.72 years in those with complete injuries and 7.19 years with incomplete injuries. Also, the survey was done by telephone or internet mail in Migliorini et al., ${ }^{1}$ in contrast to our participants being recruited when they visited hospital for annual checkup. From this method, those with higher concern of their health or with relative healthier status tend to be recruited.

Consistent with our results, the importance of EQ-5D as psychological distress predictor is well known. ${ }^{14,15} \mathrm{~A}$ number of health related quality of life measures have been developed and validated, but few have been extensively tested or used with the SCI population. ${ }^{16}$ This study showed health related quality of life was the independent predictor for psychological distress, and this result is consistent with the prior studies.

Resilience differs from health-related quality of life measured with EQ-5D. Health-related quality of life indicates the state of the measured moment, but resilience is viewed as a measure of successful stress coping ability. In other words, as resilience is not a state but one of personal characteristics, application in clinical practice with resiliency interventions can be made earlier. ${ }^{4}$ Such interventions explore resilience qualities with individuals, identify them, and nurture them. Besides the role of screening, as resilience improves with treatment, intervention effect can be assessed.

Similar to our study, previous study was done to investigate factors influencing healthy aging in patients with multiple sclerosis (MS), who were older than 55 years with MS for more than 20 years. It revealed that cognition, mental health, and resilience were interrelated factors foundational to healthy aging with multiple sclerosis. ${ }^{17}$ The patients with traumatic amputation from landmine, also sudden and devastating, were studied for revealing psychological factors. The acceptance of limb loss and their state of psychological recovery were greatly influenced by the individual's resilience characteristics. ${ }^{18}$ The patients with traumatic amputation from landmine that were also sudden and devastating were studied for revealing psychological factors. It revealed that the acceptance of limb loss and their state of psychological recovery were greatly influenced by the individual's resilience characteristics. Although both studies did not mention the role of resilience as predictor, importance to reinforce the factors including resilience was agreed.

Our limitations included use of only self-reported questionnaires to measure psychological health. This might not accurately reflect the complexity or severity of the condition. Future studies should include family-reported questionnaires or evaluation by psychiatrists. As this study is a cross-sectional design carried out with questionnaires, it is difficult to make causal inference from this study. Second, the study includes many psychological test tools: a lot of questions. Some patients did not complete the questionnaires, and were not included for analysis. Thus sampling bias can be a problem with this study. Third, it would be helpful to analyze the association of function with resilience and psychological distress level in the clinical field of rehabilitation medicine.

\section{CONCLUSION}

In this study, we examined the relations of the psychological distress level and other factors, such as SCI-specific factors, demographic characteristics and psychological factors in chronic SCI patients. Overall, psychological factors differed significantly from their psychological distress level. Clinical factors and demographic characteristics had no significant effect on psychological distress. Our results support the previously suggested importance of quality of life and resilience as predictors of psychological distress in chronic SCI patients. Future studies of interventions to manage distress according to resilience level will be useful.

\section{REFERENCES}

1. Migliorini C, Tonge B, Taleporos G. Spinal cord injury and mental health. Aust N Z J Psychiatry 2008; 42: 309314 
2. Connor KM, Davidson JR. Development of a new resilience scale: the Connor-Davidson Resilience Scale (CD-RISC). Depress Anxiety 2003; 18: 76-82

3. Catalano D, Chan F, Wilson L, Chiu CY, Muller VR. The buffering effect of resilience on depression among individuals with spinal cord injury: a structural equation model. Rehabil Psychol 2011; 56: 200-211

4. White B, Driver S, Warren AM. Resilience and indicators of adjustment during rehabilitation from a spinal cord injury. Rehabil Psychol 2010; 55: 23-32

5. Bjelland I, Dahl AA, Haug TT, Neckelmann D. The validity of the hospital anxiety and depression scale. An updated literature review. J Psychosom Res 2002; 52: 69-77

6. Reinert DF, Allen JP. The alcohol use disorders identification test: an update of research findings. Alcohol Clin Exp Res 2007; 31: 185-199

7. Rabin R, de Charro F. EQ-5D: a measure of health status from the Euroqol Group. Ann Med 2001; 33: 337343

8. Whynes DK. Correspondence between EQ-5D health state classifications and EQ VAS scores. Health Qual Life Outcomes 2008; 6: 94

9. Woolrich RA, Kennedy P, Tasiemski T. A preliminary psychometric evaluation of the Hospital Anxiety and Depression Scale (HADS) in 963 people living with a spinal cord injury. Psychol Health Med 2006; 11: 80-90

10. Schonherr MC, Groothoff JW, Mulder GA, Eisma WH. Participation and satisfaction after spinal cord injury: results of a vocational and leisure outcome study. Spinal Cord 2005; 43: 241-248
11. Shin JC, Goo HR, Yu SJ, Kim DH, Yoon SY. Depression and quality of life in patients within the first 6 months after the spinal cord injury. Ann Rehabil Med 2012; 36: $119-125$

12. Elfstrom ML, Kennedy P, Lude P, Taylor N. Conditionrelated coping strategies in persons with spinal cord lesion: a cross-national validation of the spinal cord lesion-related coping strategies questionnaire in four community samples. Spinal Cord 2007; 45: 420-428

13. Elfstrom ML, Ryden A, Kreuter M, Persson LO, Sullivan M. Linkages between coping and psychological outcome in the spinal cord lesioned: development of scl-related measures. Spinal Cord 2002; 40: 23-29

14. Alriksson-Schmidt AI, Wallander J, Biasini F. Quality of life and resilience in adolescents with a mobility disability. J Pediatr Psychol 2007; 32: 370-379

15. Whynes DK. Responsiveness of the EQ-5D to HADSidentified anxiety and depression. J Eval Clin Pract 2009; 15: 820-825

16. Andresen EM, Fouts BS, Romeis JC, Brownson CA. Performance of health-related quality-of-life instruments in a spinal cord injured population. Arch Phys Med Rehabil 1999; 80: 877-884

17. Ploughman M, Austin MW, Murdoch M, Kearney A, Fisk JD, Godwin M, Stefanelli M. Factors influencing healthy aging with multiple sclerosis: a qualitative study. Disabil Rehabil 2012; 34: 26-33

18. Ferguson AD, Richie BS, Gomez MJ. Psychological factors after traumatic amputation in landmine survivors: the bridge between physical healing and full recovery. Disabil Rehabil 2004; 26: 931-938 